\title{
Learning Task Inventories (LTIs). Exploration of Optimal Conditions to Help Students Develop, Improve and Sustain Good Study and Learning Practices
}

Stephen MacNeil, Eileen Wood, Lucia Zivcakova, Robyn Glover \& Patrick Smith, Wilfrid Laurier University

\begin{abstract}
To support students in an introductory organic chemistry course, scaffolding in the form of weekly Learning Task Inventories (LTIs) were introduced. LTIs are chapter-by-chapter lists of detailed learning tasks students are expected to master during the course. This paper describes efforts to effectively implement LTIs, the effect of differing implementations of LTIS on students' final exam grades and students' reactions to the use of LTIS.
\end{abstract}

\section{Introduction}

Students' ability to accurately monitor and assess their learning is associated with academic success (Schraw, 1998; Ku \& Ho, 2010). However, many students, even at the university level, lack the metacognitive skills and strategies that would allow them to maximize their learning (Wood, Motz \& Willoughby, 1998). In order to facilitate student learning, instructors can augment their instruction by providing both course specific content and instructional supports that scaffold students so that they can acquire the monitoring and assessment skills they need to succeed. These supports are especially important for 'feared' courses such as organic chemistry (Seymour \& Hewitt, 1997) where student anxiety can further limit students' use of appropriate learning strategies. To assist students in an introductory organic chemistry course in developing critical monitoring and self-assessment skills, we offered scaffolding support through weekly Learning Task Inventories (LTIs).

LTIs are chapter-by-chapter lists of detailed learning tasks that students are expected to master during the course. The implementation of the LTIs to the introductory Organic Chemistry I course at Wilfrid Laurier University has evolved over the past three years and culminated in the full experimental analysis presented in this paper. The nine LTIs for the 12 week course were first developed and used in 2010. Portable document formats (pdf) of the LTIs were released through the course management system at the beginning of each of nine weeks and remained available for students for the remainder of the term. We believed that these LTIs would be an excellent resource for students that could be used as check lists for weekly course review and for preparation for the midterm test and final exam, and we assumed they would be accessed early and often by most students. To our surprise, the LTIs were vastly underutilized. Figure 1 displays the percentage of students who accessed each of the LTIs throughout the term. Given that these percentages represent cumulative end-of-term data, it appears that a majority of students quickly determined that the LTIs were not worth their time. On average across the term, only $40 \%$ of students accessed the LTIs and among those who accessed the LTIs, there is no certainty that they utilized them as they were intended. 


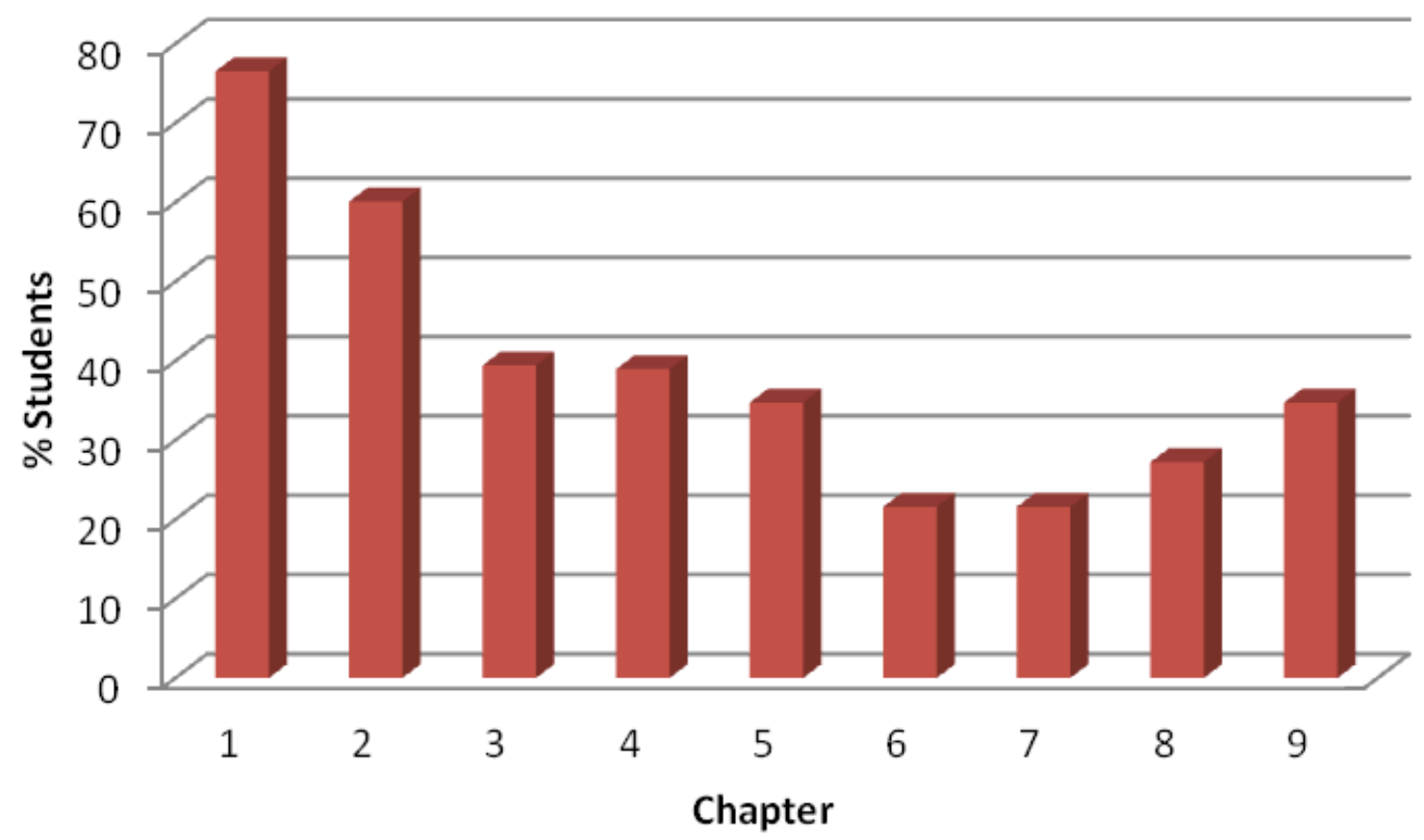

Figure 1 Percentage of students accessing LTIs by chapter (fall, 2010)

In an attempt to increase student use of LTIs in 2011, the LTIs were released as surveys through the course management system. For each learning task, students were asked to rate their ability to perform the task on a 5-point Likert scale $(1=$ cannot do this task; $5=$ can do this task very well). The LTIs were made available to students at the end of each of nine weeks, and a selective release feature of the course management system was used to effectively force students to complete each LTI in order to gain access to on-line materials for the following week. In hindsight, this was a bad idea and we have proof! Throughout the term, one of us (SM) scanned LTI results on a regular basis and noticed that, despite significant variations in level of difficulty among learning tasks, there was less and less variation in student responses as the term progressed; by the second half of the term, most learning tasks yielded response distributions that were fairly symmetrical bell curves. To test the suspicion that students were not taking the LTIs seriously, the following "learning tasks" were inserted into one of the LTIs: "state Dr. MacNeil's middle name and date of birth" and "select 1 if you are reading this". Shockingly, results for these "learning tasks" mirrored the other legitimate learning tasks, with less than half of the 222 students who completed the LTI selecting 1 in each case! With these results in hand, we came to the realization that a well designed research study was required to investigate optimal conditions for implementation of LTIs.

\section{Methods}

\section{Participants}

In the fall term of 2012, 293 (or 94\% of) students enrolled in Organic Chemistry I at Wilfrid Laurier University were recruited for the LTI research study. Willing participants signed a consent form on the first day of class. Of those participating, 186 females (69\%) and 82 males 
(31\%) reported their gender; 233 students $(86 \%)$ reported that they were $2^{\text {nd }}$ year students while 37 students $(14 \%)$ reported that they were either $3^{\text {rd }}$ or $4^{\text {th }}$ year students; and 240 students $(87 \%)$ reported that the course was required for their program whereas 37 students (13\%) reported that they were taking the course as an elective. Course enrollment by major is displayed in Figure 2. This distribution is typical of recent years.

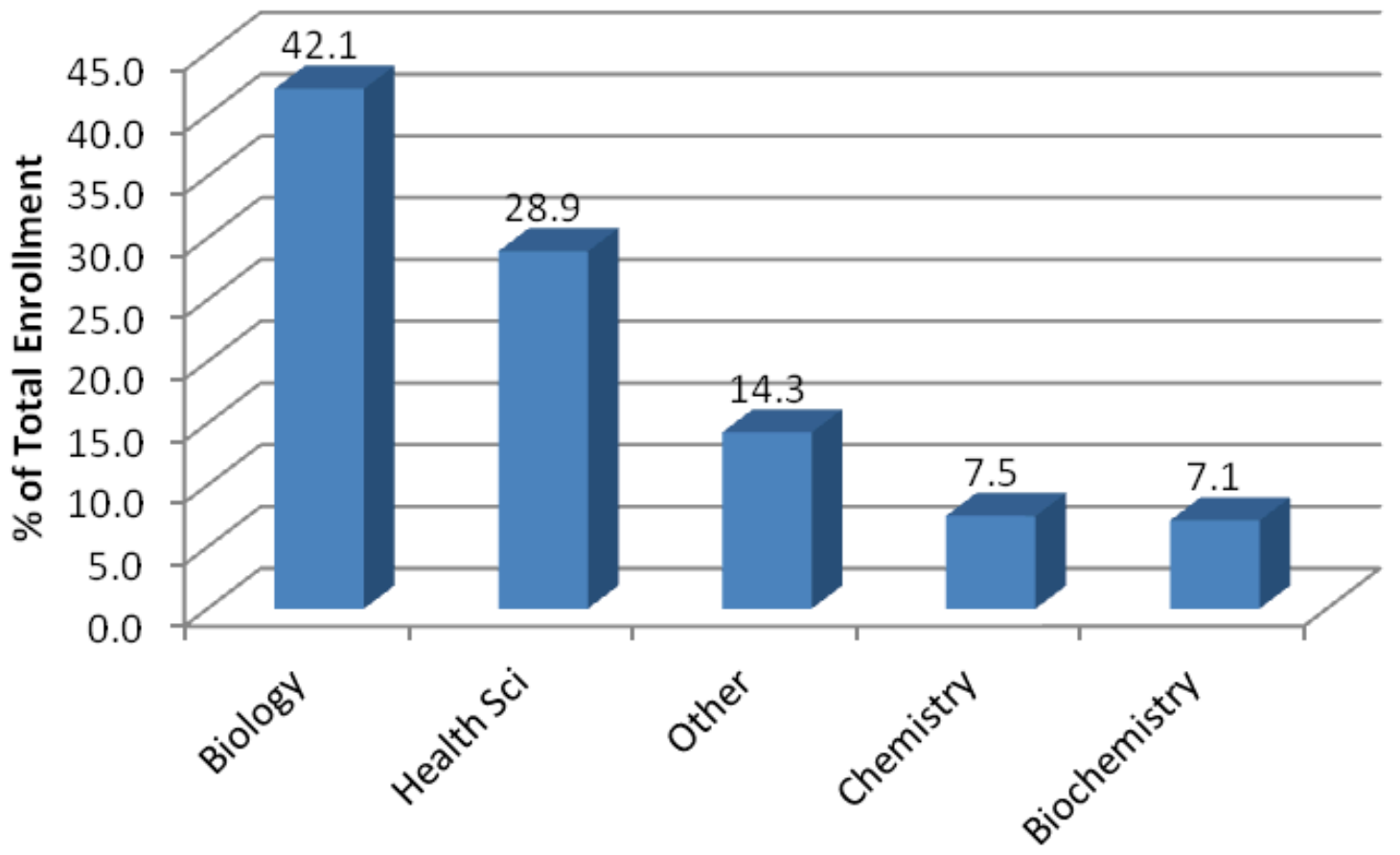

Figure 2 Organic chemistry I course enrollment by major (fall, 2012)

\section{Materials}

Students completed an introductory survey, nine weekly LTIs and an end-of-term survey. We used the introductory survey to collect demographic information (student number, age, gender, ethnicity, general chemistry grades and overall GPA, and program of study) and to gauge students' attitudes toward and expectations for the course. The end-of-term survey was used to assess student reaction to the use of LTIs. The LTIs were developed by the course instructor (SM) and made available to students at the end of each week. For each LTI, learning tasks were presented in groups of 4-6 items and for each group students answered the following questions on a 5-point Likert scale: (1) "For the previous learning tasks, how well do you think you would be able to complete the tasks?" and (2) "How difficult was the content material for these learning tasks?"

Students were assigned to one of five instructional conditions (see below) and additional materials were used to supplement the LTIs in these different treatment conditions. For example, in four conditions we introduced nine different metacognitive prompts, one per LTI, to encourage students to break away from the learning tasks and consider what they were doing. In addition, three conditions included post-LTI quizzes, consisting of five multiple choice questions related to the content of the LTI. These were used to provide further support to students and to allow them to compare perceived knowledge, as indicated by responses to the LTI, to actual 
performance. To gauge the effect of feedback, we provided two groups of students with partial or complete feedback on their quiz responses. Partial feedback revealed the correct answer without an explanation whereas complete feedback included the correct answer accompanied by a detailed explanation. All students who received the quiz also completed a post-quiz survey.

\section{Procedure}

On the first day of class, students willing to participate in the study signed a consent form and provided their email address. Students were then emailed a link and instructions for the introductory survey. Email addresses were used to randomly assign participants to one of five experimental conditions (see Table 1). Students in Condition 1 received the basic LTI only; students in Condition 2 received the LTI and the metacognitive prompts; students in Conditions 3-5 received the LTI, metacognitive prompts, the 5-question multiple choice quiz and the postquiz survey. Students in Condition 3 received no feedback on their quiz responses, students in Condition 4 received partial feedback and students in Condition 5 received complete feedback. For nine consecutive weeks of the course, excepting a break between weeks 3 and 4 for the midterm test, students were emailed a link with instructions for completing each of the nine LTIs. Following the final exam, students were emailed a link with instructions for completing the end-of-term survey.

Table 1 Variations in LTI Treatment Conditions

\begin{tabular}{llllll} 
Condition & $\mathbf{1}$ & $\mathbf{2}$ & $\mathbf{3}$ & $\mathbf{4}$ & $\mathbf{5}$ \\
LTI & $\sqrt{ }$ & $\sqrt{ }$ & $\sqrt{ }$ & $\sqrt{ }$ & $\sqrt{ }$ \\
\hline Prompt & $\mathrm{X}$ & $\sqrt{ }$ & $\sqrt{ }$ & $\sqrt{ }$ & $\sqrt{ }$ \\
\hdashline Quiz & $\mathrm{X}$ & $\mathrm{X}$ & $\sqrt{ }$ & $\sqrt{ }$ & $\sqrt{ }$ \\
\hline Quiz Feedback & $\mathrm{n} / \mathrm{a}$ & $\mathrm{n} / \mathrm{a}$ & none & partial & full \\
\hline Survey & $\mathrm{n} / \mathrm{a}$ & $\mathrm{n} / \mathrm{a}$ & $\sqrt{ }$ & $\sqrt{ }$ & $\sqrt{ }$ \\
\hline
\end{tabular}

\section{Results and Discussion}

\section{Effect of condition}

For the current study, we did not measure metacognitive skills directly. Instead, final exam grades were used as the dependent variable to test the effect of varying LTI conditions. Figure 3 depicts average final exam grades for students in each of the conditions. At first glance, it appears that students in Condition 1, those receiving the least amount of support through weekly LTIs, achieved the highest grades on the final exam. However, an analysis of variance (ANOVA) test revealed that the differences in final exam grades among treatment conditions were not statistically significant $[\mathrm{F}(4,281)=1.35, \mathrm{p}=\mathrm{n}$.s.]. This was a surprise as we had assumed greater support through quizzes and feedback would improve learning gains and translate to higher final exam grades. 


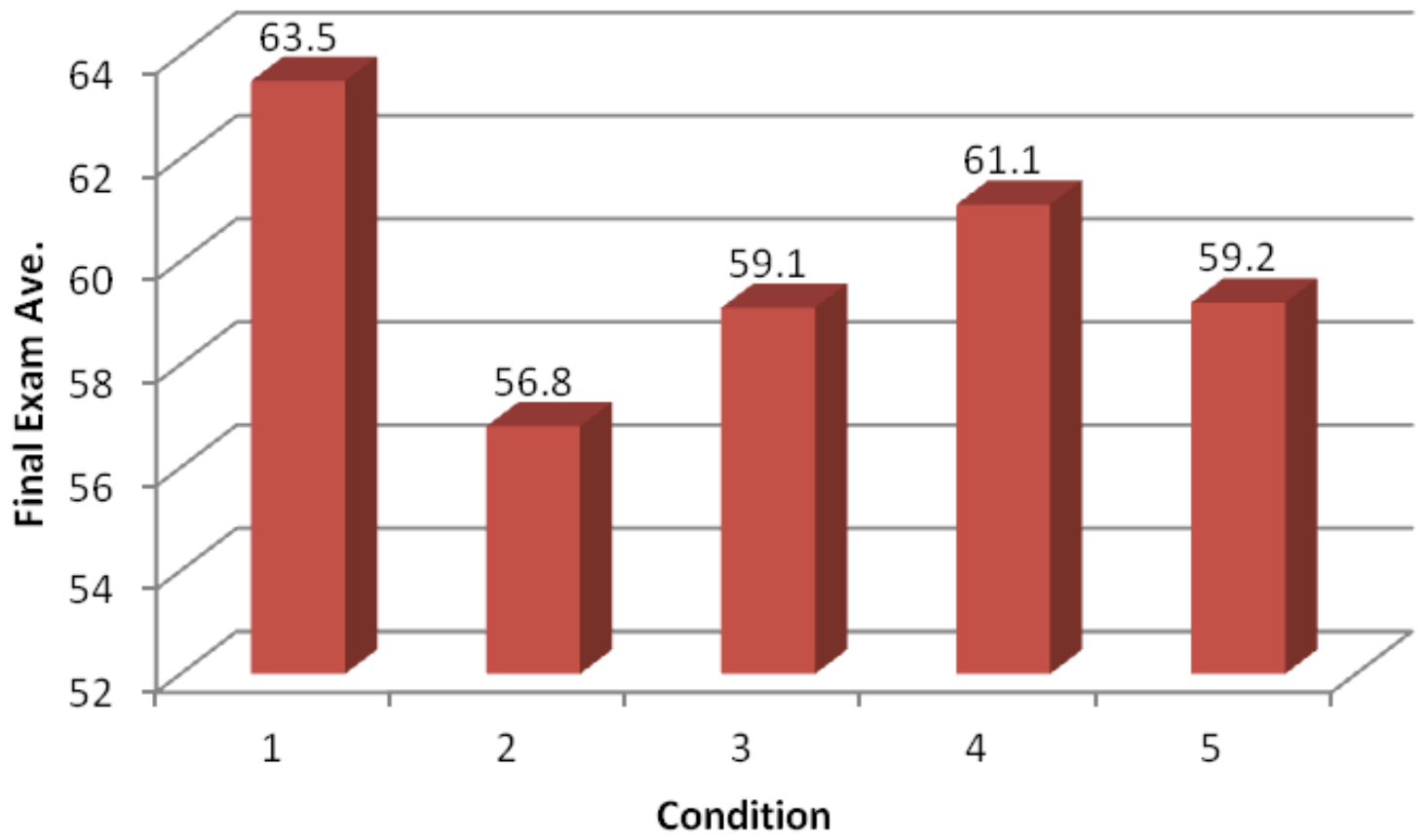

Figure 3 Final exam grades vs LTI treatment condition

\section{Effect of number of LTIs completed}

It is well known that distributed practice, the technique of dividing study efforts into frequent, relatively short study sessions, is more beneficial to student learning than is massed practice, i.e., "cramming" (Benjamin \& Tullis, 2010). A linear regression revealed that the number of LTIs completed was a significant predictor of final exam grades $[F(1,284)=33.57, p<0.001]$, with students completing more LTIs achieving higher grades. An easy criticism of this result is that better students, who would have achieved higher exam grades anyway, simply completed more LTIs.

To avoid this criticism, we ran step-wise regressions to understand the relative contribution of prior learning, as indicated by self-reported general chemistry grades (CH110 and CH111) and overall GPAs prior to the course, and the number of LTIs completed to final exam grades. Results of the step-wise regressions are summarized in Table 2. The beta weights provide a summary of the relative contributions of each of the components in the equation. By studying Table 2 it is clear that GPA and prior performance in CH110 and CH111 each predict final exam performance. However, of interest, the number of LTIs completed also predicts final exam performance and accounts for variance above and beyond that accounted for by previous GPA and chemistry course performance. 
Table 2 Step-wise regression results for prior learning vs. number of LTIS completed

\begin{tabular}{|c|c|c|c|c|c|}
\hline Variable & B & $T$ & $\mathbf{R}$ & $\mathbf{R}_{2}$ & $\Delta \mathbf{R}_{2}$ \\
\hline $\begin{array}{l}\text { Step } 1 \\
\text { o/a GPA }\end{array}$ & 4.04 & $10.68 * * *$ & 0.55 & 0.30 & 0.30 \\
\hline Step 2 & & & 0.58 & 0.34 & 0.03 \\
\hline o/a GPA & 3.68 & $9.63 * * *$ & & & \\
\hline \#LTIs completed & 2.03 & $3.69 * * *$ & & & \\
\hline Step 1 & & & 0.57 & 0.32 & 0.32 \\
\hline CH110 grade & 0.89 & $10.28 * * *$ & & & \\
\hline Step 2 & & & 0.59 & 0.34 & 0.02 \\
\hline CH110 grade & 0.84 & $9.59^{* * *}$ & & & \\
\hline \#LTIs completed & 1.54 & $2.77 * *$ & & & \\
\hline Step 1 & & & 0.49 & 0.24 & 0.24 \\
\hline CH111 grade & 0.60 & $8.47 * * *$ & & & \\
\hline Step 2 & & & 0.51 & 0.26 & 0.02 \\
\hline CH111 grade & 0.54 & $7.42 * * *$ & & & \\
\hline \#LTIs completed & 1.62 & $2.68^{* * *}$ & & & \\
\hline
\end{tabular}

Closer inspection of Table 2 reveals another salient point. The beta weights in Table 2 reveal that overall GPA is a better predictor of final exam grades in Organic Chemistry I than is the number of LTIs completed but that number of LTIs completed predicts final exam grades better than $\mathrm{CH} 110$ and $\mathrm{CH} 111$ grades do. One interpretation of this is that overall GPA reflects a student's general study skills (e.g., problem solving, time management, note taking and metacognitive skills) whereas $\mathrm{CH} 110$ and $\mathrm{CH} 111$ grades reflect a student's chemistry content knowledge, and that a student's general study skills, or "process", is more important in achieving higher grades than their content knowledge from previous relevant courses. Thus, completing more LTIs may provide a compensatory tool which may be especially advantageous for poorer students.

\section{Feedback from students}

The end-of-term survey was used to gather information on students' reactions to the use of LTIs. Figures 4 and 5 summarize students' perceptions of the impact of LTIs and LTI quizzes, respectively, on awareness of content knowledge, ease of learning course material and final grades. What is striking to us is that nearly half of the students agree that LTIs and LTI quizzes had a large impact on their awareness of what they did or did not know (88-90\% agreed they had at least some impact), but only $10-20 \%$ of students believe this contributes significantly to making course material easier to learn or to their final grades. In fact, $25-33 \%$ of students believe these resources have no impact at all on ease of learning material or final grades! When asked if completing weekly LTIs affected their study time for the midterm test and final exam, $72 \%$ and $74 \%$, respectively, responded 'NO'. In addition, $80 \%$ of students felt that use of LTIs did not change their study habits in Organic Chemistry I, and $74 \%$ did not think their study habits would change in future courses as a result of using the LTIs. However, in stark contrast to this, $72 \%$ of students said they would recommend using the LTIs in future offerings of Organic Chemistry I. Based on this feedback, we speculate that LTIs are effective at informing students about what they know and do not know, that is, they do help to develop students' metacognitive skills, but 
they may do little to support other aspects of self-regulated learning. Many students might lack the motivation to do anything about material they do not know or they may simply not know what to do with this information.

What impact did LTIs have on each of the following?

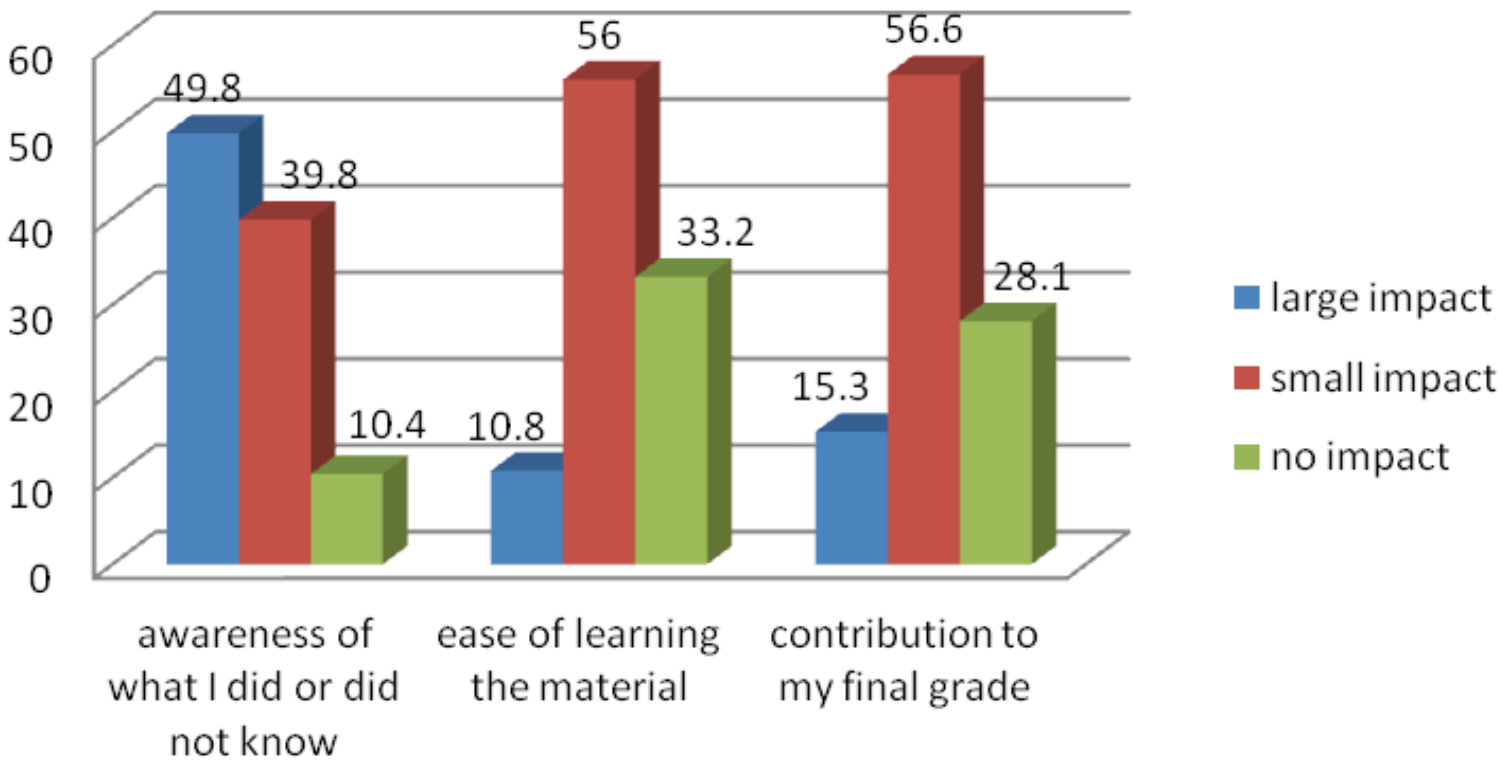

Figure 4 Student perceptions of the impact of LTIS

What impact did LTI quizzes have on each of the following?

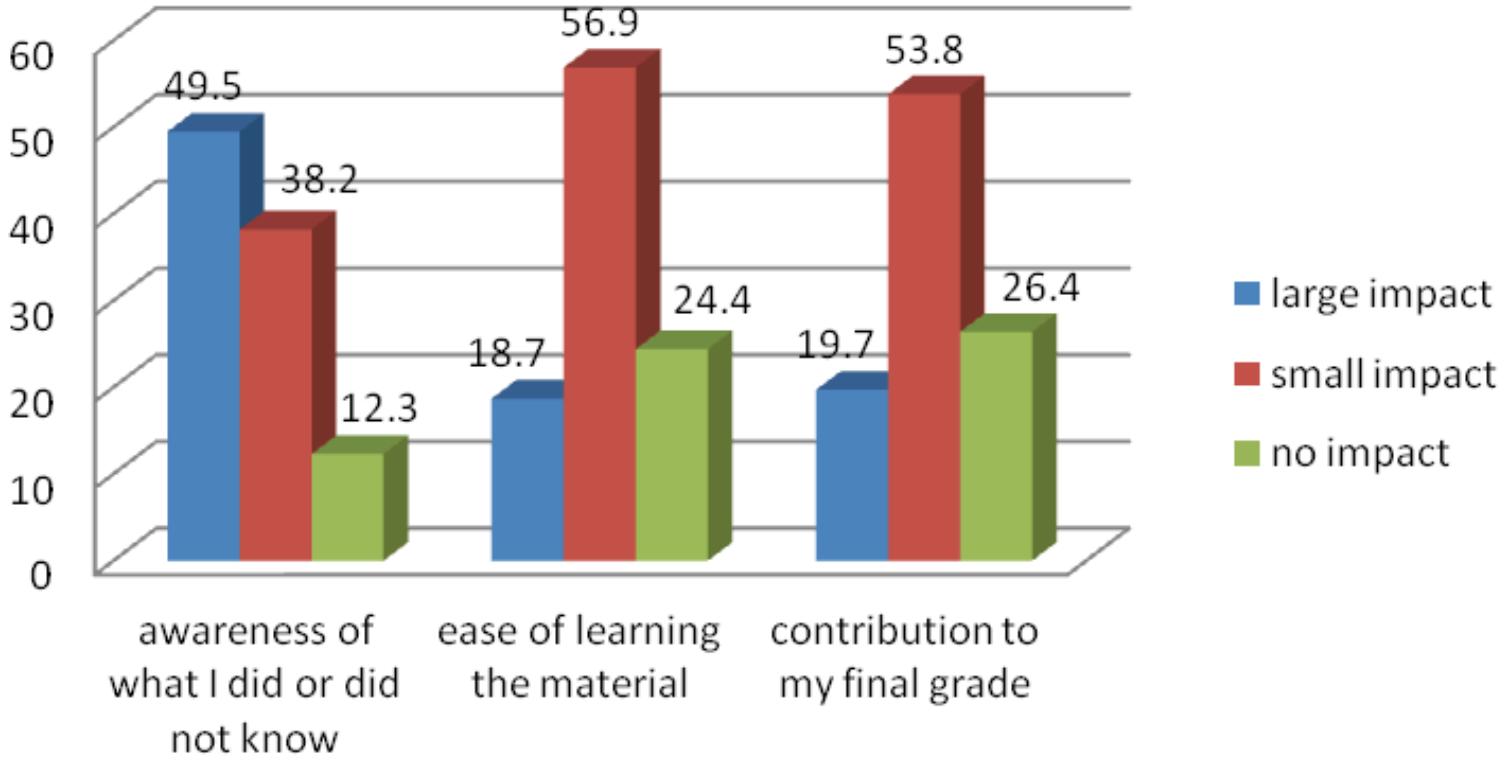

Figure 5 Student perceptions of the impact of LTI quizzes 


\section{Conclusions and Future Work}

To help students in an introductory organic chemistry class develop or improve metacognitive skills, we offered scaffolding in the form of nine weekly LTIs. We set out to determine optimal conditions for implementing the LTIs by varying the conditions to which students were exposed. The conditions reflected our perceived importance to student learning of metacognitive prompts, test taking and feedback. In the end, the various instructional supports in the conditions had no effect on final exam grades but the number of LTIs completed did, with students completing more LTIs achieving higher final exam grades. In fact, the LTIs offered advantages over and above those derived from prior learning. Feedback from students indicated the usefulness of LTIs in helping students assess what they knew and did not know but pointed to the need to support students in other aspects of self-regulated learning. Overall, these outcomes suggest that LTIs offer some benefits as an instructional tool. In future work, we will measure gains in metacognitive skills derived from LTIs using a pre-/post-test scenario, explore the effect of LTI frequency (i.e., weekly, biweekly, monthly), hold interviews with a subset of students to gain insight into how they utilize the LTIs and, in the long-term, develop scaffolding to support other aspects of self-regulated learning.

\section{References}

Benjamin, A. S.; Tullis, J. (2010). What makes distributed practice effective? Cognitive Psychology, 61, 228-247.

$\mathrm{Ku}$, K. Y. L.; Ho, I. T. (2010). Metacognitive strategies that enhance critical thinking. Metacognition Learning, 5, 251-267.

Schraw, G. (1998). Promoting general metacognitive awareness. Instructional Science, 26, 113125.

Seymour, E.; Hewitt, N. M. (1997). Talking About Leaving: Why Undergraduates Leave the Sciences; Westview Press: Boulder, CO.

\section{Biography}

Stephen MacNeil is an Associate Professor of Chemistry at Wilfrid Laurier University. He has taught introductory and intermediate courses in organic chemistry for the past 10 years, striving to convince students that organic chemistry is not the horror show it is made out to be. His research is focused on improving teaching and learning of organic chemistry in higher education.

Eileen Wood is Professor of Developmental and Instructional Psychology at Wilfrid Laurier University. Her research interests include assessing how learners of all ages acquire, retain and retrieve information. In particular, she examines these learning demands in the classroom context and in the context of different learning technologies. 
Lucia Zivcakova is enrolled in the Doctoral program in Developmental Psychology at Wilfrid Laurier University. Her research examines the impact of multi-tasking on learning and academic integrity.

Robyn Glover completed an Honours BSc Chemistry Degree at Wilfrid Laurier University in 2013. In February, 2014 she began working on a Graduate Diploma in Teaching and Learning at the University of Canterbury in Christchurch, New Zealand.

Patrick Smith completed an Honours BSc Chemistry Degree at Wilfrid Laurier University in 2013. In September, he began working toward a Bachelor of Education Degree at the University of Ottawa. 